\title{
EXISTENCE OF BLOWUP SOLUTIONS FOR NONLINEAR PROBLEMS WITH A GRADIENT TERM
}

\author{
FATEN TOUMI
}

Received 29 July 2005; Revised 7 March 2006; Accepted 25 April 2006

We prove the existence of positive explosive solutions for the equation $\Delta u+\lambda(|x|)|\nabla u(x)|$ $=\varphi(x, u(x))$ in the whole space $\mathbb{R}^{N}(N \geq 3)$, where $\lambda:[0, \infty) \rightarrow[0, \infty)$ is a continuous function and $\varphi: \mathbb{R}^{N} \times[0, \infty) \rightarrow[0, \infty)$ is required to satisfy some hypotheses detailed below. More precisely, we will give a necessary and sufficient condition for the existence of a positive solution that blows up at infinity.

Copyright (c) 2006 Hindawi Publishing Corporation. All rights reserved.

\section{Introduction and the main result}

Semilinear elliptic problems involving gradient term with boundary blowup interested many authors. Namely, Bandle and Giarrusso [1] developed existence and asymptotic behaviour results for large solutions of

$$
\Delta u+|\nabla u(x)|^{a}=f(u)
$$

in a bounded domain.

In the case $f(u)=p(x) u^{\gamma}, a>0$, and $\gamma>\max (1, a)$, Lair and Wood [7] dealt with the above equation in bounded domain and the whole space. They proved the existence of entire large solution under the condition $\int_{0}^{\infty} r \max _{|x|=r} p(x) d r<\infty$ when the domain is $\mathbb{R}^{N}$.

Recall that $u$ is a large solution on a bounded domain $\Omega$ in $\mathbb{R}^{N}$, if $u(x) \rightarrow+\infty$ as $\operatorname{dist}(x, \partial \Omega) \rightarrow 0$, and $u$ is called an entire large solution if $u$ is defined on $\mathbb{R}^{N}$ and $\lim _{|x| \rightarrow+\infty} u(x)=+\infty$.

Ghergu et al. [3] considered more general equation

$$
\Delta u+q(x)|\nabla u(x)|^{a}=p(x) f(u)
$$

where $0 \leq a \leq 2, p$ and $q$ are Hölder continuous functions on $(0, \infty)$. We note that the Keller-Osserman condition on $f$ (see $[6,8]$ ) remains the key condition for the existence for their works. 
2 Blowup solutions for semilinear problems

In the present paper, we are interested in the study of nonlinear elliptic problems with boundary blowup, of the type

$$
\begin{gathered}
\Delta u+\lambda(|x|)|\nabla u(x)|=\varphi(x, u(x)), \quad \text { in } \mathbb{R}^{N}, \\
u \geq 0, \quad u \neq 0, \\
\lim _{|x| \rightarrow+\infty} u(x)=+\infty,
\end{gathered}
$$

where $\lambda:[0, \infty) \rightarrow[0, \infty)$ is a continuous function and $\varphi$ satisfies the following hypotheses.

$\left(\mathrm{H}_{1}\right) \varphi: \mathbb{R}^{N} \times[0, \infty) \rightarrow[0, \infty)$ is measurable, continuous with respect to the second variable.

$\left(\mathrm{H}_{2}\right)$ There exist nonnegative functions $p, q$, and $f$ satisfying for each $x \in \mathbb{R}^{N}$ and $t \geq 0$,

$$
p(|x|) f(t) \leq \varphi(x, t) \leq q(|x|) f(t),
$$

where $f$ is required to satisfy.

$\left(\mathrm{H}_{3}\right) f \in \mathscr{C}^{1}([0, \infty))$ such that $f^{\prime} \geq 0, f(0)=0, f>0$ on $(0, \infty)$,

$$
\int_{1}^{\infty} \frac{1}{f(\zeta)} d \zeta=+\infty
$$

and $p, q$ are allowed to verify.

$\left(\mathrm{H}_{4}\right) p, q:(0, \infty) \rightarrow[0, \infty)$ are continuous functions satisfying

$$
\int_{0}^{1} s(1-s) q(s) d s<+\infty
$$

Clearly, we see by (1.3) that the function $p$ also satisfies (1.5).

In the sequel, we put

$$
h(r)=\int_{0}^{r} \frac{1}{K(t)}\left(\int_{0}^{t} K(s) q(s) d s\right) d t, \quad \text { for } \mathrm{r} \in[0, \infty),
$$

where $K(t):=t^{N-1} \exp \left(\int_{0}^{t} \lambda(s) d s\right)$, for each $t>0$, and we define the function $F$ on $[1, \infty)$ by

$$
F(t)=\int_{1}^{t} \frac{1}{f(\zeta)} d \zeta
$$

From the hypotheses adopted on $f$, we note that the function $F$ is a bijection from $[1, \infty)$ to $[0, \infty)$.

Our main result is the following. 
Theorem 1.1. Assume that $\left(H_{1}\right)-\left(H_{4}\right)$ hold. Moreover, assume that

$$
\int_{0}^{\infty} \frac{1}{K(t)}\left(\int_{0}^{t} K(s)(q-p)(s) f \circ F^{-1}(2 h(s)) d s\right) d t<+\infty
$$

Then problem $(P)$ has a positive entire solution if and only if

$$
\int_{1}^{\infty} \frac{1}{K(t)}\left(\int_{0}^{t} K(s) p(s) d s\right) d t=+\infty
$$

Example 1.2. Let $\alpha \geq 0$ and $\beta \in[0,1]$. Assume that for $t \geq 0, f(t)=(1+t)^{\beta} \ln (1+t)$ and $p(t)=1 / t^{\alpha}$. Then the following problem:

$$
\begin{gathered}
\Delta u+\frac{1}{1+|x|}|\nabla u(x)|=\frac{(1+u(x))^{\beta}}{|x|^{\alpha}} \ln (1+u(x)), \quad \text { in } \mathbb{R}^{N}, \\
u \geq 0, \quad u \neq 0, \\
\lim _{|x| \rightarrow+\infty} u(x)=+\infty
\end{gathered}
$$

has an explosive solution if and only if $0 \leq \alpha<2$.

Motivation for the present contribution stems from the one of Ghergu and Rădulescu [4] who considered the following problem:

$$
\begin{gathered}
\Delta u+|\nabla u(x)|=p(x) f(u), \quad \text { in } \Omega, \\
u \geq 0, \quad \text { in } \Omega,
\end{gathered}
$$

where $\Omega$ is either a smooth bounded domain or the whole space and $f$ is a nondecreasing function satisfying $f \in \mathscr{C}_{\operatorname{loc}}^{0, \alpha}(0, \infty), f(0)=0, f>0$ on $(0, \infty)$, and $\Lambda=\sup _{x \geq 1} f(x) / x<\infty$. The authors studied the existence and nonexistence of explosive solutions under the assumption that

$$
\int_{0}^{\infty} r\left(\max _{|x|=r} p(x)-\min _{|x|=r} p(x)\right) \Psi(r) d r<+\infty
$$

where $\Psi(r)=\exp \left(\Lambda /(N-2) \int_{0}^{\infty} r \min _{|x|=r} p(x) d r\right)$. More precisely, they showed in the case of $\Omega=\mathbb{R}^{N}$ that the above problem has positive solution if and only if

$$
\int_{1}^{\infty} e^{-t} t^{1-N}\left(\int_{0}^{t} e^{s} s^{N-1} \min _{|x|=s} p(x) d s\right) d t=+\infty .
$$

We remark that the condition (1.4) adopted on $f$ includes the sublinear case, $\sup _{x \geq 1} f(x) /$ $x<\infty$, studied by Ghergu and Rădulescu [4]. 
4 Blowup solutions for semilinear problems

The outline of the paper is as follows. In Section 2, we will give some auxiliary results. The comparison result obtained in Section 2, Theorem 2.6, is used in Section 3 to prove the main result of this work.

\section{Auxiliary results}

In this section, we suppose that $(A, p)$ satisfies

$\left(\mathrm{H}_{5}\right) A$ is a nonnegative continuous function on $[0, \infty)$, positive and differentiable on $(0, \infty)$, and $p:(0, \infty) \rightarrow[0, \infty)$ is continuous function satisfying

$$
\int_{0}^{1} A(s) p(s) d s<+\infty, \quad \int_{0}^{1} \frac{1}{A(t)}\left(\int_{0}^{t} A(s) p(s) d s\right) d t<+\infty .
$$

For any given continuous function $\psi$ on $(0, \infty)$, we put

$$
h_{\psi}(r)=\int_{0}^{r} \frac{1}{A(t)}\left(\int_{0}^{t} A(s) \psi(s) d s\right) d t, \quad \text { for } \mathrm{r} \in[0, \infty)
$$

We consider the following problem:

$$
\begin{gathered}
\frac{1}{A}\left(A u^{\prime}\right)^{\prime}=p(t) f(u), \quad \text { in }(0, \infty), \\
A u^{\prime}(0):=\lim _{t \rightarrow 0^{+}} A(t) u^{\prime}(t)=0, \quad u(0)=\alpha \geq 1 .
\end{gathered}
$$

We state the following.

Theorem 2.1. Under the hypotheses $\left(H_{3}\right)$ and $\left(H_{5}\right)$, the problem (2.3) has a positive solution $u \in \mathscr{C}([0, \infty)) \cap \mathscr{C}^{1}((0, \infty))$. Further, on $[0, \infty)$,

$$
\alpha+f(\alpha) h_{p}(r) \leq u(r) \leq F^{-1}\left(F(\alpha)+h_{p}(r)\right)
$$

Proof. Let $\left(u_{k}\right)_{k \geq 0}$ be the sequence of functions defined on $[0, \infty)$ by $u_{0}(r)=\alpha$ and

$$
u_{k+1}(r)=\alpha+\int_{0}^{r} \frac{1}{A(t)}\left(\int_{0}^{t} A(s) p(s) f\left(u_{k}(s)\right) d s\right) d t, \quad \forall k \in \mathbb{N} .
$$

Clearly, we have for each $k \in \mathbb{N}, t \rightarrow u_{k}(t)$ is a nondecreasing function on $[0,+\infty)$.

By induction, we prove that $\left(u_{k}\right)_{k \geq 0}$ is a nondecreasing sequence.

Since the function $f$ is nondecreasing, we obtain by (2.5) that for each $k \geq 0$,

$$
u_{k}^{\prime}(t) \leq f\left(u_{k}(t)\right) \frac{1}{A(t)} \int_{0}^{t} A(s) p(s) d s, \quad t \geq 0 .
$$

That is,

$$
\frac{u_{k}^{\prime}(t)}{f\left(u_{k}(t)\right)} \leq \frac{1}{A(t)} \int_{0}^{t} A(s) p(s) d s, \quad t \geq 0
$$


Then

$$
\int_{0}^{r} \frac{u_{k}^{\prime}(t)}{f\left(u_{k}(t)\right)} d t \leq \int_{0}^{r} \frac{1}{A(t)}\left(\int_{0}^{t} A(s) p(s) d s\right) d t, \quad r \geq 0 .
$$

It follows that for each $r \geq 0$,

$$
F\left(u_{k}(r)\right)-F(\alpha)=\int_{\alpha}^{u_{k}(r)} \frac{1}{f(\zeta)} d \zeta \leq h_{p}(r) .
$$

So

$$
u_{k}(r) \leq F^{-1}\left(F(\alpha)+h_{p}(r)\right), \quad r \geq 0
$$

Then the sequence $\left(u_{k}\right)_{k \geq 0}$ converges and the function $u=\sup _{k \in \mathbb{N}} u_{k}$ is finite and satisfies for each $r \geq 0$,

$$
u(r)=\alpha+\int_{0}^{r} \frac{1}{A(t)}\left(\int_{0}^{t} A(s) p(s) f(u(s)) d s\right) d t
$$

So, $u \in \mathscr{C}([0, \infty)) \cap \mathscr{C}^{1}((0, \infty))$. Thus $u$ is a solution of the problem (2.3). Moreover, from the monotonicity of $f$ and (2.10), we obtain (2.4).

Remark 2.2. The solution of problem (2.3) satisfying (2.4) is bounded if and only if

$$
\int_{0}^{+\infty} \frac{1}{A(t)}\left(\int_{0}^{t} A(s) p(s) d s\right) d t<+\infty
$$

Example 2.3. Let $A(t)=t^{\delta}$ for $t \in[0, \infty)$, where $\delta \geq 0$. Assume that for $t>0, p(t)=$ $1 / t^{\mu}(1+t)^{\nu-\mu}$, with $\mu<\min (2,1+\delta)$ and $\nu \in \mathbb{R}$. Let $a, b \geq 0$ such that $a+b>0, \beta \geq 0$, and $0 \leq \alpha \leq 1$, set $f(t)=\left(a t^{\alpha}+b\right) \ln \left(1+t^{\beta}\right)$ for $t \in[0, \infty)$, then the problem

$$
\begin{gathered}
\frac{1}{A}\left(A u^{\prime}\right)^{\prime}=\frac{1}{t^{\mu}(1+t)^{\nu-\mu}} f(u(t)), \quad \text { in }[0, \infty), \\
A u^{\prime}(0)=0, \quad u(0)=u_{0} \geq 1
\end{gathered}
$$

has a positive solution $u \in \mathscr{C}([0, \infty)) \cap \mathscr{C}^{\infty}((0, \infty))$. Moreover $u$ is bounded if and only if $\delta>1$ and $\mu<2<\nu$.

Corollary 2.4. Assume $\left(H_{3}\right)$ and $\left(H_{5}\right)$ hold. Assume moreover that $\left(H_{6}\right)$ holds, for all $c>0$, there exists $k>0$ such that for all $x, y \in[0, c],|f(x)-f(y)| \leq k|x-y|$. Then the problem (2.3) has a unique positive solution $u \in \mathscr{C}([0, \infty)) \cap \mathscr{C}^{1}((0, \infty))$ satisfying (2.4).

Proof. Existence follows from Theorem 2.1.

Now, let us prove the uniqueness. Let $u$ and $v$ be positive solutions of the problem (2.3). Then for each $a>0$ and $r \in[0, a]$, we have

$$
|u(r)-v(r)| \leq \int_{0}^{r} \frac{1}{A(t)}\left(\int_{0}^{t} A(s) p(s)|f(u(s))-f(v(s))| d s\right) d t .
$$


Since $u$ and $v$ are continuous, it follows that there exists $c>0$ such that $u(r), v(r) \in[0, c]$ for each $r \in[0, a]$.

So, by hypothesis $\left(\mathrm{H}_{6}\right)$ and Fubini theorem, we obtain that

$$
|u(r)-v(r)| \leq k \int_{0}^{r}\left[A(s) p(s)\left(\int_{s}^{a} \frac{1}{A(t)} d t\right)\right]|u(s)-v(s)| d s .
$$

By Gronwall's lemma, we deduce that $u(r)=v(r)$ on $[0, a]$. This completes the proof.

Corollary 2.5. Let $\lambda:[0, \infty) \rightarrow[0, \infty)$ be a continuous function and suppose that $f$ and $(A, p)$ satisfy, respectively, $\left(H_{3}\right)$ and $\left(H_{5}\right)$. Then the problem

$$
\begin{gathered}
\frac{1}{A}\left(A u^{\prime}\right)^{\prime}+\lambda(u)\left(u^{\prime}\right)^{2}=p(t) f(u), \quad \text { in }(0, \infty), \\
A u^{\prime}(0)=0, \quad u(0)=\alpha \geq 1
\end{gathered}
$$

has a positive solution $u \in \mathscr{C}([0, \infty)) \cap \mathscr{C}^{1}((0, \infty))$.

Proof. Let $\rho:[0, \infty) \rightarrow[0, \infty)$ be the function defined by $\rho(t)=\int_{0}^{t} \exp \left(\int_{0}^{\xi} \lambda(s) d s\right) d \xi$. It is clear that $\rho$ is a bijection from $[0, \infty)$ to itself. Put $v=\rho(u)$. Then $v$ satisfies the following problem:

$$
\begin{aligned}
& \frac{1}{A}\left(A v^{\prime}\right)^{\prime}=p(t) g(v), \quad \text { in }(0, \infty), \\
& A v^{\prime}(0)=0, \quad v(0)=\rho(\alpha) \geq 1,
\end{aligned}
$$

where the function $g$ is defined on $[0, \infty)$ by $g \circ \rho=\rho^{\prime} f$. Clearly, $g$ satisfies $\left(\mathrm{H}_{3}\right)$. Hence by Theorem 2.1, the above problem has a solution $v$ belonging to $\mathscr{C}([0, \infty)) \cap \mathscr{C}^{1}((0, \infty))$. Therefore, $u=\rho^{-1}(v)$ is a solution of the problem (2.16). This completes the proof.

Now, we will give a comparison result. For this aim, we suppose in what follows that

(i) $(A, p)$ and $(B, q)$ satisfy $\left(\mathrm{H}_{5}\right), p \leq q$, and $B / A$ is nondecreasing function,

(ii) $f$ and $g$ satisfy $\left(\mathrm{H}_{3}\right)$ with $0 \leq g \leq f$.

For each $c \geq 1$, we define on $[0,+\infty)$ the function

$$
m_{c}(r):=G^{-1}\left(G(c)+h_{q}(r)\right)
$$

where $h_{q}$ is the function defined by (2.2) and $G^{-1}$ is the inverse of the function $G(t)=$ $\int_{1}^{t} 1 / g(\zeta) d \zeta$.

Theorem 2.6. Assume that the assumptions (i) and (ii) are satisfied. Then for any $\beta \geq 1$ satisfying

$$
\int_{0}^{\infty} \frac{1}{B(t)}\left(\int_{0}^{t} B(s)(q-p)(s) g\left(m_{\beta}(s)\right) d s\right) d t<+\infty
$$


there exists $\alpha>\beta$ such that problems

$$
\begin{aligned}
\frac{1}{A}\left(A v^{\prime}\right)^{\prime} & =p(t) f(v), \quad \text { in }[0, \infty), \\
A v^{\prime}(0) & =0, \quad v(0)=\alpha>1, \\
\frac{1}{B}\left(B w^{\prime}\right)^{\prime} & =q(t) g(w), \quad \text { in }[0, \infty), \\
A w^{\prime}(0) & =0, \quad w(0)=\beta \geq 1
\end{aligned}
$$

have positive continuous solutions satisfying

$$
v \geq w, \quad \text { in }[0, \infty) \text {. }
$$

Proof. By Theorem 2.1, for any $\alpha>\beta \geq 1$, problems (2.20) have positive solutions $v$ and $w$ satisfying the integral equations

$$
\begin{aligned}
& v(r)=\alpha+\int_{0}^{r} \frac{1}{A(t)}\left(\int_{0}^{t} A(s) p(s) f(v(s)) d s\right) d t, \quad r \geq 0, \\
& w(r)=\beta+\int_{0}^{r} \frac{1}{B(t)}\left(\int_{0}^{t} B(s) q(s) g(w(s)) d s\right) d t, \quad r \geq 0 .
\end{aligned}
$$

Let $\alpha>\beta \geq 1$. We intend to show that if the constant $\alpha$ is sufficiently large, more precisely

$$
\alpha>\beta+\left(\int_{0}^{\infty}\left(\int_{0}^{t} \frac{B(s)}{B(t)}(q-p)(s) g\left(m_{\beta}(s)\right) d s\right) d t\right)
$$

then we have

$$
v(r) \geq w(r), \quad r \geq 0 .
$$

Using (ii) and the fact that $B / A$ and $f$ are nondecreasing functions on $[0, \infty)$, we obtain

$$
\begin{aligned}
w(r)= & \beta+\int_{0}^{r}\left(\int_{0}^{t} \frac{B(s)}{B(t)} q(s) g(w(s)) d s\right) d t \\
\leq & \beta+\int_{0}^{r} \frac{1}{B(t)}\left(\int_{0}^{t} B(s)(q-p)(s) g(w(s)) d s\right) d t \\
& +\int_{0}^{r} \frac{1}{A(t)}\left(\int_{0}^{t} A(s) p(s) f(w(s)) d s\right) d t .
\end{aligned}
$$

On the other hand, by (2.4), we have

$$
w(r) \leq G^{-1}\left(G(\beta)+\int_{0}^{r} \frac{1}{A(t)}\left(\int_{0}^{t} A(s) q(s) d s\right) d t\right)=m_{\beta}(r) .
$$


8 Blowup solutions for semilinear problems

By (2.19) and (2.23), we obtain

$$
\begin{aligned}
w(r) & -\int_{0}^{r}\left(\int_{0}^{t} \frac{A(s)}{A(t)} p(s) f(w(s)) d s\right) d t \\
& \leq \beta+\int_{0}^{r}\left(\int_{0}^{t} \frac{B(s)}{B(t)}(q-p)(s) g\left(m_{\beta}(s)\right) d s\right) d t \\
& <\alpha=v(r)-\int_{0}^{r} \frac{1}{A(t)}\left(\int_{0}^{t} \frac{A(s)}{A(t)} p(s) f(v(s)) d s\right) d t .
\end{aligned}
$$

Then using a standard comparison theorem [9, Theorem VI, page 17], we obtain (2.21).

\section{Proof of the main result}

Proof of Theorem 1.1. Recall that for each $t>0, K(t):=t^{N-1} \exp \left(\int_{0}^{t} \lambda(s) d s\right)$.

Necessity. We will proceed by contradiction. Suppose that (1.9) fails and let $u$ be an entire large solution of problem $(P)$. Let

$$
v(x):=\int_{1}^{u(x)+1} \frac{1}{f(\zeta)} d \zeta .
$$

Define the spherical mean of $v$ by

$$
\bar{v}(r):=\frac{1}{w_{N} r^{N-1}} \int_{|x|=r} v(x) d \sigma_{x}
$$

where $w_{N}$ denotes the surface of the unit sphere in $\mathbb{R}^{N}$.

Since $u$ is a positive entire large solution of $(P)$, it follows by (1.4) that $v$ is positive and $\lim _{|x| \rightarrow \infty} v(x)=+\infty$.

By [2, Section 1, Proposition 6], we obtain

$$
\Delta \bar{v}=\bar{v}^{\prime \prime}+\frac{N-1}{r} \bar{v}^{\prime}=\overline{\Delta v}
$$

So

$$
\Delta \bar{v}+\lambda(|x|) \nabla \bar{v} \leq \frac{1}{w_{N} r^{N-1}} \int_{|x|=r} \Delta v(x)+\lambda(|x|)|\nabla v(x)| d \sigma_{x}
$$

By computation, we have on the ball

$$
\begin{aligned}
\Delta v(x)+\lambda(|x|)|\nabla v(x)|= & \frac{1}{f(u(x)+1)} \Delta u(x)+\left(\frac{1}{f}\right)^{\prime}(u(x)+1)|\nabla u(x)|^{2} \\
& +\frac{1}{f(u(x)+1)} \lambda(|x|)|\nabla u(x)| .
\end{aligned}
$$


Using the fact that $f^{\prime} \geq 0$, we obtain

$$
\begin{aligned}
\Delta \bar{v}+\lambda(|x|) \nabla \bar{v} & \leq \frac{1}{w_{N} r^{N-1}} \int_{|x|=r} \frac{1}{f(u(x)+1)}(\Delta u(x)+\lambda(|x|)|\nabla u(x)|) d \sigma_{x} \\
& \leq \frac{1}{w_{N} r^{N-1}} \int_{|x|=r} \frac{1}{f(u(x)+1)} p(|x|) f(u(x)) d \sigma_{x} \leq p(r) .
\end{aligned}
$$

That is,

$$
\bar{v}^{\prime \prime}+\frac{N-1}{r} \bar{v}^{\prime}+\lambda(r) \bar{v}^{\prime} \leq p(r)
$$

Then

$$
\left(r^{N-1} \exp \left(\int_{0}^{r} \lambda(s) d s\right) \bar{v}^{\prime}\right)^{\prime} \leq r^{N-1} \exp \left(\int_{0}^{r} \lambda(s) d s\right) p(r) .
$$

Integrating (3.8) yields for each $r \geq r_{0}>0, \bar{v}(r) \leq \bar{v}\left(r_{0}\right)+\int_{0}^{r} 1 / K(t)\left(\int_{0}^{t} K(s) p(s) d s\right) d t$. Thus $\bar{v}$ is bounded, contradiction. It follows that $(P)$ has no positive large solution.

Sufficiency. Suppose that (1.9) holds. We will use the comparison result given by Theorem 2.6 for $A(t)=B(t)=K(t)=t^{N-1} \exp \left(\int_{0}^{t} \lambda(s) d s\right), p, q$, and $f$ satisfying, respectively, $\left(\mathrm{H}_{4}\right)$ and $\left(\mathrm{H}_{3}\right)$.

Let $\beta \geq 1$. Put for $r \geq 0$,

$$
m_{\beta}(r):=F^{-1}(F(\beta)+h(r))
$$

where $h$ is the function defined by (1.6).

First, we claim that

$$
\int_{0}^{\infty} \frac{1}{K(t)}\left(\int_{0}^{t} K(s)(q-p)(s) f\left(m_{\beta}(s)\right) d s\right) d t<+\infty .
$$

In fact, by (1.3) and (1.9), there exists $0<r_{0}<+\infty$ such that

$$
F(\beta)<\int_{0}^{r_{0}} \frac{1}{K(t)}\left(\int_{0}^{t} K(s) q(s) d s\right) d t=h\left(r_{0}\right)
$$

Then

$$
\begin{aligned}
\int_{0}^{r_{0}} & \frac{1}{K(t)}\left(\int_{0}^{t} K(s)(q-p)(s) f\left(m_{\beta}(s)\right) d s\right) d t \\
& <\int_{0}^{r_{0}} \frac{1}{K(t)}\left(\int_{0}^{t} K(s)(q-p)(s) f \circ F^{-1}\left(2 h\left(r_{0}\right)\right) d s\right) d t \\
& <f \circ F^{-1}\left(2 h\left(r_{0}\right)\right) \int_{0}^{r_{0}} \frac{1}{K(t)}\left(\int_{0}^{t} K(s) q(s) d s\right) d t<+\infty .
\end{aligned}
$$


On the other hand, by (1.8), we obtain

$$
\begin{aligned}
& \int_{r_{0}}^{\infty} \frac{1}{K(t)}\left(\int_{0}^{t} K(s)(q-p)(s) f\left(m_{\beta}(s)\right) d s\right) d t \\
& \quad<\int_{r_{0}}^{\infty} \frac{1}{K(t)}\left(\int_{0}^{t} K(s)(q-p)(s) f \circ F^{-1}(2 h(s)) d s\right) d t<+\infty .
\end{aligned}
$$

This yields (3.10).

Thus by Theorem 2.6, there exists $\alpha>\beta$ such that the problems

$$
\begin{aligned}
\frac{1}{K}\left(K v^{\prime}\right)^{\prime} & =p(t) f(v), \quad \text { in }[0, \infty), \\
K v^{\prime}(0) & =0, \quad v(0)=\alpha>1, \\
\frac{1}{K}\left(K w^{\prime}\right)^{\prime} & =q(t) f(w), \quad \text { in }[0, \infty), \\
K w^{\prime}(0) & =0, \quad w(0)=\beta \geq 1
\end{aligned}
$$

have positive solutions satisfying $v \geq w$ in $[0, \infty)$.

Now, for all $k \geq 0$, we consider the problem

$$
\begin{gathered}
\Delta u_{k}+\lambda(|x|)\left|\nabla u_{k}(x)\right|=\varphi\left(x, u_{k}(x)\right), \quad \text { in } B(0, k), \\
u_{k}(x)=v(k), \quad \text { on } \partial B(0, k) .
\end{gathered}
$$

It is clear that $w$ and $v$ are positive sub- and supersolutions of $\left(P_{k}\right)$. Then the problem $\left(P_{k}\right)$ has at least a positive solution $u_{k}$ and

$$
w(|x|) \leq u_{k}(x) \leq v(|x|), \quad \text { in } B(0, k), \forall k \geq 1 .
$$

Now, by [5, Theorem 14.3], the sequence $\left(\nabla u_{k}\right)_{k}$ is bounded on every compact set in $\mathbb{R}^{N}$. Consequently, the sequence $\left(u_{k}\right)_{k}$ is bounded and equicontinuous on each compact of $\mathbb{R}^{N}$. Therefore, by Ascoli-Arzèla theorem, the sequence $\left(u_{k}\right)_{k}$ has a uniformly convergent, subsequence $\left(u_{k}^{1}\right)_{k}$ in $\mathscr{C}(\overline{B(0,1)})$. Setting $u^{1}=\lim _{k \rightarrow+\infty} u_{k}^{1}$. Then $\left(\varphi\left(\cdot, u_{k}^{1}\right)\right)_{k}$ converges uniformly to $\varphi\left(\cdot, u^{1}\right)$ and so $\left(\Delta u_{k}^{1}+\lambda(|x|)\left|\nabla u_{k}^{1}(x)\right|\right)_{k}$ converges uniformly to $\varphi\left(\cdot, u^{1}\right)$ on $B(0,1)$.

Then, using the fact that $(\Delta+\lambda \nabla)$ is a closed operator, we conclude that $u^{1}$ satisfies $(P)$ in $B(0,1)$.

Similarly, the sequence $\left(u_{k}^{1}\right)_{k}$ has a uniformly convergent sequence $\left(u_{k}^{2}\right)_{k}$ on $B(0,2)$ and let $u^{2}=\lim _{k \rightarrow+\infty} u_{k}^{2}$. Using the same arguments as above, we claim that $u^{2}$ satisfies $(P)$ in $B(0,2)$. Further, we have $u^{2}=u^{1}$ on $B(0,1)$.

Repeating this procedure, we construct a sequence $\left(u^{n}\right)_{n}$ satisfying $(P)$ in $B(0, n)$ and $u^{n+1}=u^{n}$ on $B(0, n)$, for all $n$. The sequence $\left(u^{n}\right)_{n}$ converges in $L_{\mathrm{loc}}^{\infty}\left(\mathbb{R}^{N}\right)$ to the function $u$ given by $u(x)=u^{n}(x)$ on $B(0, n)$. 
Using (3.15), we obtain $w \leq u^{n} \leq v$ in $B(0, n)$, for all $n \geq 1$. Letting $n$ to $+\infty$, it follows that $w \leq u \leq v$ in $\mathbb{R}^{N}$ and $u$ satisfies the equation

$$
\Delta u+\lambda(|x|)|\nabla u(x)|=\varphi(x, u(x)), \quad \text { in } \mathbb{R}^{N} .
$$

By (1.9) and Remark 2.2, we obtain $\lim _{|x| \rightarrow \infty} w(x)=+\infty$.

Consequently, $u$ is a positive entire large solution of problem $(P)$.

\section{Acknowledgments}

I express my sincere gratitude to Professor Habib Mâagli for his guidance and the useful discussions. Thanks go to the referees for valuable comments and useful remarks on the paper.

\section{References}

[1] C. Bandle and E. Giarrusso, Boundary blow-up for semilinear elliptic equations with nonlinear gradient terms, Advances in Differential Equations 1 (1989), 133-150.

[2] R. Dautray, J.-L. Lions, et al., Analyse mathématique et calcul numérique pour les sciences et les techniques. Vol. 2: L'opérateur de Laplace, Coll. C.E.A, Masson, Paris, 1987.

[3] M. Ghergu, C. Niculescu, and V. Rădulescu, Explosive solutions of elliptic equations with absorption and non-linear gradient term, Proceedings of the Indian Academy of Sciences. Mathematical Sciences 112 (2002), no. 3, 441-451.

[4] M. Ghergu and V. Rădulescu, Nonradial blow-up solutions of sublinear elliptic equations with gradient term, Communications on Pure and Applied Analysis 3 (2004), no. 3, 465-474.

[5] D. Gilbarg and N. S. Trudinger, Elliptic Partial Differential Equations of Second Order, Springer, Berlin, 1977.

[6] J. B. Keller, On solutions of $\Delta u=f(u)$, Communications on Pure and Applied Mathematics 10 (1957), 503-510.

[7] A. V. Lair and A. W. Wood, Large solutions of semilinear elliptic equations with nonlinear gradient terms, International Journal of Mathematics and Mathematical Sciences 22 (1999), no. 4, 869883.

[8] R. Osserman, On the inequality $\Delta u \geqslant f(u)$, Pacific Journal of Mathematics 7 (1957), 16411647.

[9] W. Walter, Differential and Integral Inequalities, Ergebnisse der Mathematik und ihrer Grenzgebiete, Band 55, Springer, New York, 1970.

Faten Toumi: Département des Mathématiques, Faculté des Sciences de Tunis,

Campus Universitaire, 2092 Tunis, Tunisia

E-mail address: faten.toumi@fsb.rnu.tn 


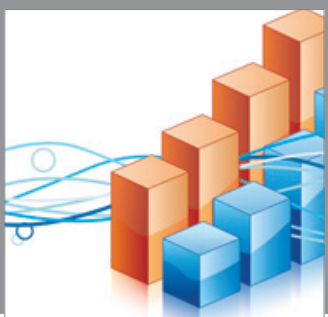

Advances in

Operations Research

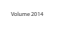

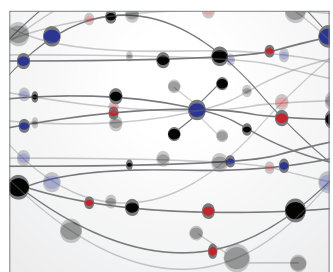

\section{The Scientific} World Journal
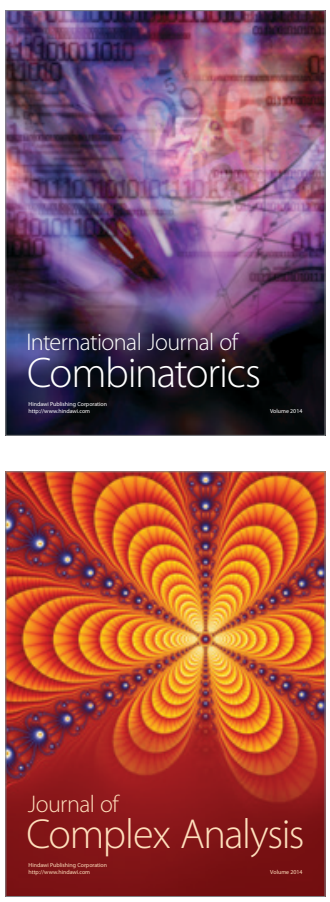

International Journal of

Mathematics and

Mathematical

Sciences
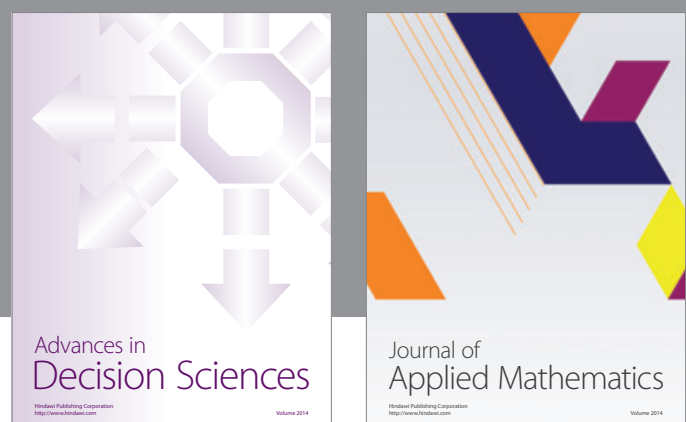

Journal of

Applied Mathematics
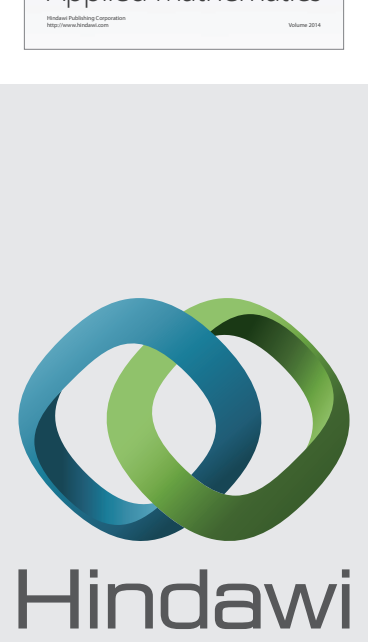

Submit your manuscripts at http://www.hindawi.com
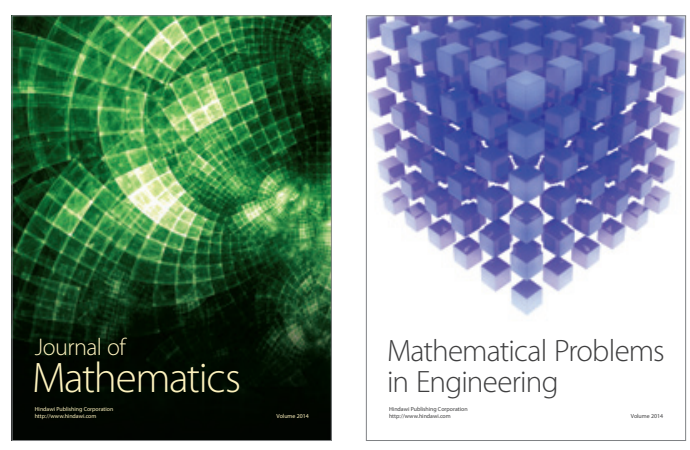

Mathematical Problems in Engineering
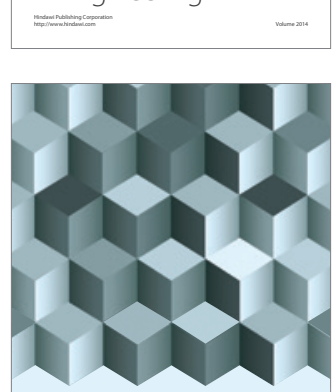

Journal of

Function Spaces
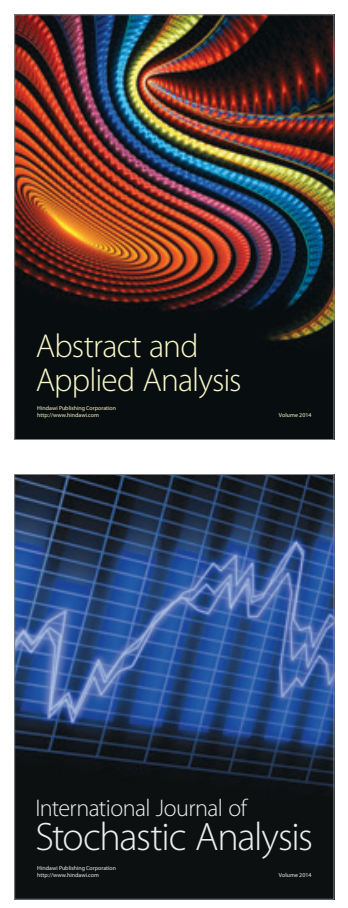

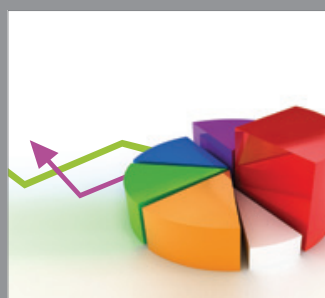

ournal of

Probability and Statistics

Promensencen
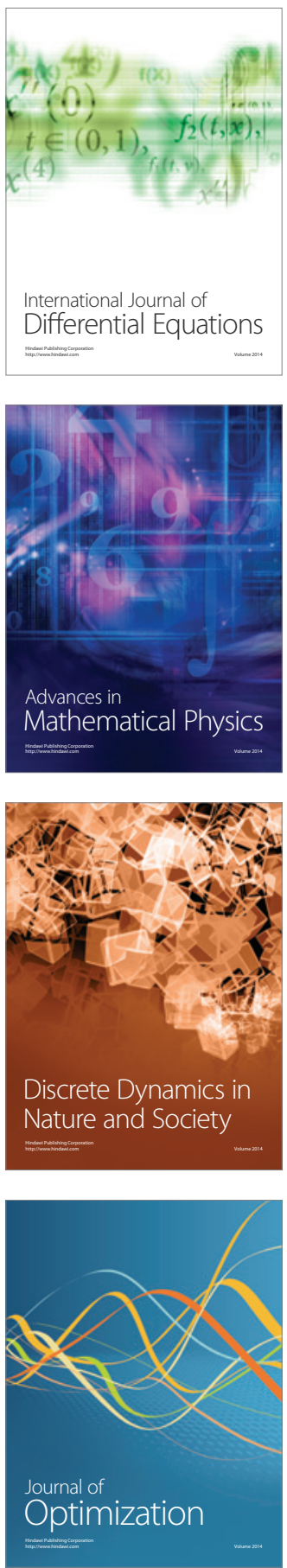